\title{
Inicio de alimentación complementaria y riesgo de enfermedad celíaca y alergia alimentaria. ¿De qué evidencia disponemos?
}

\author{
Complementary feeding and risk \\ of celiac disease and food allergy. \\ What does the evidence say?
}

\begin{abstract}
Celiac disease is a chronic systemic disorder mediated by immune and autoimmune phenomena, triggered by ingestion of gluten and related prolaminas in genetically susceptible individuals. Food allergy is an adverse, immune mediated, reproducible reaction to a foodstuff. Recommendations on complementary feeding have varied along time and also vary in different countries, depending on their cultures, socioeconomic resources and dietary habits. To date the scientific evidence is not clear about how the nature and quantity of foods given to a child and the forms in which they are given influence health and futures development in the child. Recent publications question some basic criteria of pediatric feeding, like the protective role of maternal milk and the benefit of postponing the introduction of some frequently allergenic foods; they suggest that the age of introduction of gluten and other potentially allergenic foodstuffs do not modify the risk of developing celiac disease or food allergy and that breast feeding does not protect from these two conditions. For these reasons we have considered relevant to review the available evidence on these matters on celiac disease and food allergy.

Key words: celiac disease, food allergy, complementary feeding.
\end{abstract}

\section{INTRODUCCIÓN}

La Enfermedad Celíaca (EC) es una enfermedad crónica que se manifiesta como un desorden sistémico mediado por fenómenos inmunes y autoinmunes, gatillado por el gluten y prolaminas relacionadas, en individuos genéticamente susceptibles. Su inicio es generalmente gradual, existiendo un período de latencia variable de meses a años entre el inicio de la ingesta de gluten y la aparición de la enfermedad. Su prevalencia es 1-3\% en la población general (1). La frecuencia de la EC ha aumentado consistentemente en el mundo a lo largo de los últimos 10-20 años, fenómeno que también se observa en nuestro país. La segunda Encuesta Nacional de Salud chilena mostró que $0,75 \%$ de la población >15 años tiene anticuerpos IgA-antitransglutaminasa (TTG) positivos y la prevalencia de la EC confirmada por biopsia intestinal se estima en $\sim 0,6 \%(2)$.

La evidencia actual disponible no es suficiente para entender por qué se ha producido este aumento. Las mejores
Elizabeth Navarro $(1,2)$

Magdalena Araya (2)

(1) Programa de Formación de Especialista en Gastroenterología Pediátrica Universidad de Chile. Hospital de niños Exequiel González Cortés. Departamento de Pediatría y Cirugía Infantil Campus Sur, Facultad de Medicina, Universidad de Chile.

(2) Laboratorio de Gastroenterología, Unidad de Nutrición Humana, Instituto de Nutricion y Tecnologia de los Alimentos INTA Universidad de Chile, Santiago, Chile.

Dirigir la correspondencia a: Profesora

Magdalena Araya INTA, Universidad de Chile Macul 5540, Santiago, Chile Email:maraya@inta.uchile.c www.inta.cl

Este trabajo fue recibido el 28 de Enero de 2016 y aceptado para ser publicado el 2 de Julio de 2016. herramientas diagnósticas y la mayor conciencia acerca de la enfermedad en los profesionales de la salud y en la población general influyen en el fenómeno, pero algunos estudios sugieren además que la prevalencia real de la EC ha aumentado (3). En el siglo pasado, la sociedad occidental presentó una tendencia general al aumento de las patologías relacionadas al sistema inmune, incluyendo fenómenos de alergia y autoinmunidad. La teoría de la higiene es una atractiva manera de explicar estos problemas pero no ha podido ser demostrada (4). Es probable que el mejor conocimiento acerca de la microbiota intestinal humana en el futuro permita entender al menos parte de los aspectos etiopatogénicos hoy día poco claros (5).

La alergia alimentaria (AA) es una reacción adversa, inmuno-mediada y reproducible a un alimento (6), siendo más común en niños, principalmente lactantes y preescolares (7). Al igual que la EC, su frecuencia ha aumentado ostensiblemente durante el siglo pasado y las causas del aumento 
tampoco están claras. La mayoría de los autores invocan las características de "la vida moderna" de la sociedad occidental entre los factores que serían determinantes de estos cambios (3). Entre los alergenos más comunes están la proteína de la leche de vaca, huevo, soya, pescados, mariscos y frutos secos. Su prevalencia no está bien establecida, estimándose que 2-6\% de los niños estaría afectado (8), cifra que iría en aumento tanto en países en desarrollo como en los desarrollados. En Chile existe evidencia que sugiere que en los menores de 1 año de nivel socio económico medio-alto la prevalencia es alrededor de 3\% (9).

Las recomendaciones acerca de la alimentación complementaria han variado a lo largo del tiempo y también varían en los distintos países según sus culturas, medios económicos y hábitos alimentarios. Hasta el momento la evidencia científica no logra explicar cómo la naturaleza de los alimentos entregados, la cantidad de éstos o la forma de darlos, influyen en la salud y desarrollo a futuro del niño.

Hoy en día existe una activa discusión acerca del momento más seguro para introducir el gluten y algunos otros alimentos. Estudios publicados recientemente han puesto en duda algunos criterios básicos de la alimentación en pediatría, como el rol protector de la lactancia materna (LM) o el beneficio de retardar la introducción de ciertos alimentos considerados alergénicos a la dieta del niño. La nueva evidencia sugiere que la edad de introducción del gluten no modificaría el riesgo de desarrollar la EC y que la LM no confiere protección para EC ni para alergia alimentaria. Por estas razones hemos considerado de interés evaluar la evidencia disponible en el tema en estas dos condiciones, la enfermedad celíaca y la alergia alimentaria.

\section{Alimentación complementaria del lactante}

La Organización Mundial de la Salud (OMS) describe la alimentación complementaria como todo alimento, sólido o líquido, que recibe el niño, diferente de la leche materna; esta definición incluye a las fórmulas lácteas, como una manera de enfatizar la importancia de la lactancia natural. Sin embargo, en esta revisión nos referiremos a alimentación complementaria como todo alimento distinto a la LM o fórmula láctea, siguiendo las recomendaciones de alimentación complementaria de la Sociedad Europea de Gastroenterología, Hepatología y Nutrición Pediátrica (ESPGHAN) y de la Rama de Nutrición de la Sociedad Chilena de Pediatría.

\section{¿Hay una edad óptima para introducir} los sólidos a la dieta del lactante?

No existe consenso acerca de la edad óptima para introducir los alimentos sólidos a la dieta. Lanigan y cols, realizaron una revisión sistemática (2001) focalizada específicamente en la edad óptima para introducir sólidos en niños recibiendo leche materna y/o fórmula. Su conclusión fue que no hay evidencias suficientes para cambiar el criterio de la OMS, que recomienda iniciarlos entre los 4 y 6 meses (10). El comité de nutrición de la ESPGHAN, en 2008 consideró conveniente no iniciar la alimentación con sólidos antes de las 17 ni después de las 26 semanas de vida. Se basó en las necesidades nutricionales del niño, habilidades de desarrollo, datos publicados en relación a la edad de introducción de sólidos y las consecuencias en la salud a futuro (11). Por su parte, la Academia Americana de Pediatría el año 2014 también recomendó la introducción de sólidos a partir de los 6 meses (12). En Chile, las nuevas recomendaciones para la alimentación normal del niño menor de 2 años, publicadas por la Rama de Nutrición en
2013, establecieron también la edad de 6 meses como inicio de la alimentación complementaria, con un rango entre los 5 y 6 meses para su comienzo (13).

Si bien parece haber consenso global en establecer que la edad de inicio de la alimentación sólida sea entre los 4 y 6 meses de edad, no hay evidencias que muestren el momento óptimo de introducir determinados alimentos, existiendo amplias variaciones en los diversos países. Por ejemplo, la leche de vaca (no modificada) en Dinamarca, Suecia y Canadá se introduce entre los 9 y 10 meses, mientras que en varios países se recomienda no darla antes del año (11). Para el pescado y el huevo, la recomendación más frecuente es iniciarlos entre los 4-6 meses, aunque en algunos países se sugiere esperar hasta los 9-12 meses (12). En Chile, se recomienda introducir el pescado entre los 6-7 meses y el huevo entre los 9-10 meses, independiente del riesgo de atopia o antecedentes familiares de alergia (13).

\section{Aspectos biológicos y de neurodesarrollo}

Un factor relevante a tomar en cuenta para fijar criterios de cómo alimentar al lactante se refiere al desarrollo de la función digestiva. A los 4 meses de edad ésta es suficientemente madura para digerir y absorber nutrientes distintos a la leche de la dieta. La exposición a sólidos implica una transición desde una alimentación rica en grasas a otra alta en carbohidratos. Esto se asocia a respuestas hormonales, que favorecen la adaptación de las funciones digestivas a la naturaleza de las comidas ingeridas, aumentando la cantidad y/o actividad de ciertas enzimas (11).

La función renal es otro factor a considerar. Durante los primeros tres meses de vida el lactante mejora la filtración glomerular, que le permite mayor tolerancia al agua y solutos, aunque ésta es aun limitada. La excreción y reabsorción tubular pueden ser relativamente bajas todavía, pero si la alimentación es adecuada la función renal del lactante es suficiente. Por el contrario, si el lactante recibe alimentos con elevada carga de solutos, se favorece el balance hídrico negativo, lo que podría ocurrir si se utilizan fórmulas no adaptadas o si la introducción de alimentos sólidos es precoz. Los riñones maduran morfológica y funcionalmente a lo largo del primer año de la vida (14).

El desarrollo psicomotor alcanzado también es importante. A los 6 meses los niños ya se pueden sentar con apoyo, sacar la comida que se le ofrece en una cuchara y usar su musculatura masticatoria. Además, se inicia la erupción dentaria y se incrementan las percepciones sensoriales (olfato, visión, tacto y gusto por los alimentos). A los 8 meses la lengua tiene mayor flexibilidad y los niños pueden tragar porciones más grandes y grumosas. Entre los 9 y 12 meses ya se han adquirido destrezas manuales suficientes para tomar alimentos con las manos y -con algunas limitaciones- logran compartir el momento de la comida junto el resto de la familia (11). Si a los 10 meses no se han introducido aún alimentos sólidos, se describe mayor riesgo de trastornos de la conducta alimentaria en el futuro (11).

\section{Aspectos nutricionales}

La leche materna es capaz de cubrir las necesidades energéticas y nutricionales del niño hasta los 6 meses de edad; a partir de entonces se hace insuficiente y es necesario complementar la alimentación para asegurar un buen crecimiento y desarrollo. Mantener la lactancia exclusiva después de los 6 meses de vida puede resultar en deficiencia de macronutrientes (proteínas), algunos micronutrientes (principalmente hierro y 
zinc) y algunas vitaminas (principalmente $A$ y D) $(11,13)$. Tan importante como evitar las deficiencias es el evitar los aportes excesivos, fundamentalmente de energía y proteínas, que se asocian con mayor riesgo de obesidad en la edad escolar (11, $15,16)$. Este fenómeno se observa con mayor frecuencia en lactantes alimentados con fórmulas (15); hoy se sugiere supervisar las cantidades que ingieren ya que tienden a beber volúmenes mayores que los niños alimentados al pecho, lo que hace posible que la cantidad de macro- y micro- nutrientes y vitaminas resulten excesivas (11).

\section{LACTANCIA MATERNA Y RIESGO DE EC}

Hay varios estudios que sugieren que la $L M$ reduciría el riesgo de presentar EC a futuro, sin embargo, la mayoría son retrospectivos y sólo unos pocos son randomizados (17). En relación al estudio prospectivo realizado por Norris (18), se observó que los niños que introdujeron el gluten antes de los 3 meses tuvieron 5 veces mayor riesgo de desarrollar autoinmunidad que los que lo hicieron a los 4-6 meses, pero no hubo efecto de la LM sobre el desarrollo de la autoinmunidad. Ackobeng (2006) publicó una revisión sistemática y meta-análisis de los efectos de la leche materna y riesgo de EC (19) y concluyó que la leche materna ofrece protección contra esta enfermedad, calculando que los niños con LM al momento de la introducción del gluten reducirían en 52\% el riesgo de desarrollar EC, comparado con sus pares sin lactancia al momento de iniciar el gluten. Los niños nacidos durante la epidemia de EC sueca, asociada a la introducción temprana de gluten, tuvieron mayor frecuencia (3\%) de EC a los 12 años, mientras que aquellos nacidos después de la epidemia disminuyeron la frecuencia a $0.75 \%$ (Cl 0.60-0.93) (20). Estos datos sugieren una "ventana de oportunidad" entre los $4 \mathrm{y}$ 6 meses en que se debería introducir el gluten en pequeñas cantidades y mientras aún el niño tiene LM (11).

Los mecanismos por los cuales la LM protegería del desarrollo de EC no son claros. Se ha propuesto que la lactancia al iniciar el gluten limitaría la cantidad ingerida, disminuyendo así la probabilidad de desarrollo de autoinmunidad e inflamación (19). Ivarsson y cols describieron que al momento de iniciar los sólidos los niños celíacos recibían mayor cantidad de harinas que sus pares controles (21). La LM también actuaría previniendo las infecciones gastrointestinales del lactante. Estas aumentan transitoriamente la permeabilidad de la mucosa y la expresión de la transglutaminasa tisular, favoreciendo la producción de los péptidos deamidados de gliadina que participan en el inicio de la cascada inmune que gatilla la enfermedad (22). Mincheva-Nilsson y cols han propuesto que la IgA de la LM podría disminuir la respuesta al gluten ingerido, ya que podría aglutinar el antígeno en la superficie de la mucosa, evitando su paso a través de epitelio. Por último, por sus propiedades inmunomoduladoras, la LM podría ejercer un efecto supresor en subgrupos de células T específicas (23).

Entre los estudios que rechazan el efecto protector de la LM está el de Welander y cols, quienes no encontraron relación entre la duración de la lactancia, edad de inicio de la introducción del gluten y desarrollo posterior de EC. El resultado se mantuvo cuando se corrigió por la presencia de infecciones digestivas al momento de iniciar el gluten (24). Roberts y cols, tampoco encontraron diferencias en la incidencia acumulada de EC entre niños que recibieron o no LM (25). Recientemente, Vriezinga y cols (1) evaluaron niños amamantados y con riesgo genético de EC, administrándoles $100 \mathrm{mg}$ de gluten desde los 4 o desde los 6 meses de edad y no encontraron diferencias significativas en la frecuencia de aparición de EC a los 3 años de edad. Concluyeron que la $L M$ no previene EC a los 3 años de edad. Cualquiera sea el efecto de la $L M$, se debe también aclarar si produciría un efecto persistente o sólo un retraso en la edad de aparición y diagnóstico de la enfermedad (17).

\section{Edad de introducción del gluten y riesgo de enfermedad celíaca}

Estudios observacionales han mostrado que el desarrollo de tolerancia oral al gluten se inicia tempranamente en la vida. Los datos de Ivarsson revelaron que hay tres factores interactuando: la cantidad de gluten ingerida, edad de introducción y presencia de LM al momento de la introducción (20). En una cohorte noruega (26), el inicio de ingesta de gluten después de los 6 meses se asoció a un mayor riesgo de EC, lo cual concuerda con estudios previos de Norris (18). Hasta ahora la recomendación general ha sido iniciar el gluten entre los 4 y 6 meses, preferentemente mientras el niño aún esté con lactancia materna $(11,13,19,27)$. Hay que tomar en cuenta que estas recomendaciones se basan en un número limitado de estudios y los resultados han sido obtenidos en poblaciones de características muy distintas. Nuevos estudios prospectivos, intervencionales, recientemente terminados has sido realizados en Europa (PREVENTCD) y sus primeros resultados empiezan a aparecer en la literatura (28). El estudio de Vriezinga (1) se evaluó niños con riesgo de EC (por tener un familiar de primer grado con EC y portar HLA-DQ2 y/o DQ8), y se les separó aleatoriamente en los que recibieron $200 \mathrm{mg}$ de gluten o bien placebo ( $2 \mathrm{~g}$ de lactosa) a partir de las 16 semanas de vida, diariamente, durante 8 semanas; luego no recibieron gluten hasta los 6 meses, recomendándose LM durante todo el periodo de estudio; 994 niños cumplieron el protocolo. La incidencia acumulada de EC a los 3 años fue $5,2 \%$, no observándose diferencias entre ambos grupos. No se encontró relación entre el desarrollo de EC y la duración de la $\mathrm{L} M$, ni con el mantenimiento de ésta durante la introducción del gluten. Esto ha llevado a proponer que aunque la nutrición temprana es claramente un factor relevante en la salud futura, se requiere más evidencia para establecer el mejor momento para introducir el gluten y prevenir EC (29).

En el estudio multicéntrico CELIPREV (30) se evaluó una cohorte de recién nacidos con riesgo de EC (al menos un familiar de primer grado con EC), seleccionados y aleatorizados a iniciar la ingesta de gluten a los 6 meses o a los 12 meses. Durante el primer año de vida se recopiló información sobre infecciones intestinales y consumo diario de gluten. A los 15 meses se hizo genotipificación, los anticuerpos antigliadina y antitransglutaminasa (TTG) se determinaron a $\operatorname{los} 2,3,5,8$ y 10 años, en 553 niños. A los 2 años, los niños que recibieron gluten desde los 6 meses de edad, habían desarrollado EC en un porcentaje significativamente más alto (12 vs. 5\%) que aquellos que lo iniciaron a los 12 meses. Sin embargo, a los 5 y a los 10 años las diferencias entre grupos desaparecieron y la LM no se asoció con el desarrollo de EC. En la cohorte prospectiva TEDDY (27) se estudió el genotipo HLA- DQ2 y DQ8 en una cohorte de recién nacidos en Finlandia, Alemania, Suecia y Estados Unidos. Se estudiaron 6436 niños, siguiéndose la presencia de TTG cada 3 meses. Si dos muestras eran positivas los niños eran evaluados por un gastroenterólogo para decidir la necesidad de biopsia intestinal. La media de seguimiento fue 5 años; $12 \%$ de los niños presentaron TTG positiva y $5 \%$ desarrolló EC. La introducción del gluten antes de las 17 o 
después de las 26 semanas de vida no se asoció con mayor riesgo de positividad de los TTG o de EC.

En resumen, la evidencia reciente sugiere que la edad de introducción del gluten no modificaría el riesgo de desarrollar la EC y no parece sustentar la hipótesis de una ventana de edad que propicie el desarrollo de tolerancia oral. Sin duda es necesario continuar investigando el tema para sacar conclusiones definitivas.

\section{INTRODUCCIÓN DE ALERGENOS Y DESARROLLO DE ALERGIA ALIMENTARIA}

Las enfermedades alérgicas son otras de las condiciones que están aumentando su prevalencia en el mundo, tanto en los países desarrollados como en desarrollo (31). La literatura disponible es controversial, en buena parte debido a que las grandes diferencias existentes en las metodologías utilizadas, los criterios para hacer los diagnósticos y a las poblaciones estudiadas. Más que optar por una posición definida, creemos importante poner en el tapete de discusión lo controversial de los resultados publicados y la falta de evidencia para instaurar nuevas normas de introducción de los alergenos en la dieta. Un ejemplo de esta situación es la Guía 2015 WAO (World Allergy Organization) (32); en ella se indica que "la evidencia disponible aun no permite concluir que la suplementación con probióticos disminuye el riesgo de desarrollar alergia en lo niños". Sin embargo, el mismo documento "sugiere que se usen probióticos a) en las mujeres embarazadas con alto riesgo de tener un niño alérgico, b) en mujeres lactantes cuyo niño tiene riesgo de desarrollar alergia y c) en lactantes con alto riesgo de desarrollar alergia. Todas estas recomendaciones son condicionales y están apoyadas en evidencia de nivel bajo".

Otro punto relevante es que, hasta ahora, la evidencia disponible no permite apoyar recomendaciones diferentes en la introducción de la alimentación complementaria para los niños de mayor riesgo de padecer alergia, entendiendo como riesgo el tener un familiar de primer grado con historia de alergia. Por otro lado, las dietas hechas por la madre durante el embarazo o lactancia no tendrían efecto en la aparición de alergia en el niño (33-35). En la cohorte del estudio PIFA del Reino Unido, se seleccionó un grupo de pacientes con AA diagnosticada mediante desafío doble-ciego, placebocontrol, observándose que la introducción de alimentación complementaria antes de las 16 semanas de edad se asociaba a mayor frecuencia de aparición de alergia alimentaria. Así, se sugirió que la LM sería un factor de protección para la alergia a la proteína de la leche de vaca, cuando ésta era introducida mientras el niño aún consume leche materna (26). Sin embargo en otro estudio, (una cohorte de 13.000 niños en que se evaluaron factores de riesgo asociados al desarrollo de alergia a la proteína de leche de vaca), los resultados fueron al revés de lo esperado (36). En los lactantes expuestos a la proteína durante las primeras 2 semanas de vida la incidencia de alergia fue significativa menor, en comparación con los lactantes expuestos después de los 4-6 meses (36). Los resultados se interpretaron como que el contacto temprano con la proteína favorecería el desarrollo de tolerancia.

Una mención especial merece la alergia al maní, frecuente en algunos países y menos frecuente en Chile. Du Toit (37) y cols compararon la prevalencia de alergia al maní entre niños judíos que vivían en el Reino Unido o Israel, encontrando una prevalencia 10 veces mayor en el Reino Unido. La única diferencia que se detectó fue que las familias del Reino Unido no exponían al niño al maní durante los primeros 3 años de vida, mientras que los israelíes que lo empezaban a consumir más temprano y en mayor cantidad. Aunque no indiquen cómo y porqué ocurre, estos datos apoyan la idea que la edad, la forma y la cantidad del alimento al momento de la introducción de ciertos alimentos en la dieta sí influyen en el desarrollo posterior de alergia.

En nuestro país, las recomendaciones de Guías de diagnóstico y manejo de APLV vigentes (8) plantean que para prevenir la alergia el principal factor es mantener la LM exclusiva hasta los 4-6 meses e iniciar la alimentación complementaria a partir de esta edad, sin retrasar la introducción de aquellos alimentos habitualmente considerados potencialmente alergénicos.

En resumen, la evidencia disponible aún no es concluyente. El valor de la $L M$ en la nutrición y desarrollo del niño es enorme y no parece razonable reducir su participación al fenómeno alérgico, por lo que las indicaciones de lactancia materna actualmente vigentes debieran permanecer. Independiente del momento de introducción de un nuevo alimento, parece prudente que la exposición a él sea repetida frecuentemente y en pequeñas cantidades, de manera de favorecer la tolerancia oral. Una de las ideas que recoge progresivamente más adeptos, es que al introducir la alimentación complementaria se debe incluir la mayor diversidad de alimentos posibles, ya que los estudios recientes sugieren que en el primer año de vida esta práctica se asociada a menor frecuencia posterior de asma, alergia alimentaria o sensibilización a alimentos (38).

\section{CONCLUSIONES}

Si bien los estudios aparecidos recientemente acerca de la introducción del gluten en la dieta del lactante no algunas recomendaciones actuales, tampoco las invalidan de manera categórica, dejando claro que la evidencia disponible es aún insuficiente para establecer nuevas recomendaciones.

A la fecha, se desconoce la edad óptima de introducción de alimentos potencialmente alergénicos, la forma y cantidad más adecuada de administrarlos, tanto en población general como en niños de riesgo. Mantener la LM exclusiva hasta los 6 meses, por todos los beneficios que conlleva, e iniciar la introducción de sólidos entre los 4-6 meses, por los aspectos nutricionales y de desarrollo descritos, parece ser adecuado.

La idea de una ventana de protección otorgada por la lactancia materna y los mecanismos por los cuales actuaría constituye una hipótesis atractiva pese a que no todos los estudios la apoyen. Deberemos esperar nuevas evidencias que permitan esclarecer estos temas.

\section{RESUMEN}

La Enfermedad Celíaca (EC) es una enfermedad sistémica crónica mediada por fenómenos inmunes y autoinmunes, gatillado por la ingesta de gluten y prolaminas relacionadas, en individuos genéticamente susceptibles. La alergia alimentaria (AA) es una reacción adversa, inmuno-mediada, reproducible a un alimento. Las recomendaciones acerca de la alimentación complementaria han variado a lo largo del tiempo y también varían en los distintos países según sus culturas, medios económicos y hábitos alimentarios. Hasta el momento la evidencia científica no entrega certeza en cómo la naturaleza de los alimentos entregados, la cantidad de éstos o la forma de darlos, influyen en la salud y desarrollo a futuro del niño. Estudios publicados recientemente han puesto en duda algunos criterios básicos de la alimentación en pediatría, como el rol protector de la lactancia materna o el beneficio de 
retardar la introducción de ciertos alimentos frecuentemente alergénicos, sugiriendo que la edad de introducción del gluten y otros alimentos potencialmente alergénicos no modificaría el riesgo de desarrollar la EC ni la AA y que la lactancia materna no confiere protección para $E C$ ni para $A A$. Por estas razones hemos considerado de interés evaluar la evidencia disponible acerca de la EC y la AA.

Palabras clave: enfermedad celíaca, alergia alimentaria, alimentación complementaria.

\section{BIBLIOGRAFÍA}

1. Vriezinga $S$, Auricchio $R$, Castillejo $G$ et al. Randomized feeding intervention in infants at high risk for celiac disease. N Engl J Med. 2014; 371 (14): 1304-15.

2. MINSAL. Encuesta Nacional de Salud ENS Chile: 2009 2010.

3. Catassi C, Kryszak D, Bhatti B, Sturgeon C, Helzlsouer K, Clipp SL, et al. Natural history of celiac disease autoimmunity in a USA cohort followed since 1974. Ann Med. 2010;42:530-8.

4. Bach JF. The effect of infections on susceptibility to autoimmune and allergic diseases. N Engl J Med. 2002 Sep 19;347(12):911-20.

5. Olivares $M$, Neef $A$, Castillejo G, Palma GD, Varea $V$, Capilla $A$, et al. The HLA-DQ2 genotype selects for early intestinal microbiota composition in infants at high risk of developing coeliac disease. Gut. 2015;64(3):406-17. PubMed PMID: 24939571.

6. Carrard A, Rizzuti D, Sokollik C. Update on food allergy. Allergy. 2015; 70 (12): 1511-20.

7. Anagnostou K, Stiefel G, Brough H, Du Toit G, Lack G, Fox A. Active Management of food allergy: an emerging concept. Arch Dis Child 2015; 100: 386-390.

8. MINSAL. Guía Clínica Alergia a la Proteína de Leche de Vaca. 2012.

9. Rossel M., Araya M. Food allergy in infancy. Rev Med Clin Las Condes 2011;22(2):184-9..

10. Lanigan J, Bishop JA, Kimber AC, et al. Systematic review concerning the age of introduction of complementary food to the healthy full-term infant. Eur J Clin Nutr. 2001; 55 : 309-20.

11. Agostoni $C$, Decsi T, Fewtrell M et al. Complementary Feeding: A Commentary by the ESGHAN Commite on Nutrition. J Pediatr Gastroenterol Nutr. 2008; 46 (1): 99-110.

12. Ribes C, Dalmau J, Moreno J, Díaz J, Castillejo de Villasante $G$, Polanco I. Introduction of gluten in infant diet. An Pediatr (Barc). 2015;83(5):565-72.

13. Castillo C, Balboa P, Torrejón C, Bascuñán $K$, Uauy $R$. Normal feeding of child under 2 years of age. Rev Chil Pediatr. 2013;84(5):565-72.

14. Lázar A, Martín B. Normal Feeding of infant. Diagnostictherapeutic protocols of Gastroenterology, Hepatology and Pediatric Nutrition. ESPGHAN-AEP 2010: p 287-95.

15. Huh S, Rifas-Shiman S, Taveras E, Oken E, Gillman $M$. Timing of Solid Food Introduction and Risk of Obesity in Preschool-Aged Children. Pediatrics. 2011; 127: e544e551.

16. Koletzko B, Bhuttab Z, Caic W et al. Compositional Requirements of Follow-up Formula for use in infancy: Recommendations of an International Expert Group Coordinated by the early Nutrition Academy. Ann Nutr Metab. 2013; 62: 44-54.

17. Ludvigsson J, Fasano A. Timing of introduction of gluten and Celiac Disease Risk. Ann Nutr Metab. 2012; 60 (suppl
2): 22-9.

18. Norris J, Barriga K, Hoffenberg E et al. Risk of celiac diseaseautoinmunity and Timing of gluten Introduction in the Diet of infants at Increased Risk of Disease. JAMA. 2005; 293 (19): 2344-51.

19. 19 Akobeng A, Ramanan A, Buchan I, Heller R. Effect of breast feeding on risk of coeliac disease: a systematic review and meta-analysis of observational Studies. Arch Dis Child. 2006; 91: 39-43.

20. Ivarsson A, Myléus A, Norström F et al. Prevalence of Childhood Celiac Disease and changes in Infant Feeding. 2013; 131: e687-e694.

21. Ivarsson A, Hernell $O$, Stenlund $H$ et al. Breast-feeding protects against celiac disease. Am J Clin Nutr. 2002; 75: 914-21.

22. Sollid L. Breastmilk against coeliac disease. Gut. 2002; 51: 767-768.

23. Mincheva-Nilsson L, Hammarstrom M, Juto P et al. Human milk contains proteins that stimulate and supress T lymphocite proliferation. Clin Exp Immunol. 1990; 79: 463-469.

24. Welander A, Honeymann $M$, Hoffenberg $E$ et al. Infectious disease and risk of later celiac disease in childhood. Pediatrics. 2010: 125: e530-e536.

25. Roberts S, Williams J, Meddings D et al. Perinatal risk factors and coeliac disease in children and young adults: a record linkage study. Aliment Pharmacol Ther. 2009; 29: 222-31.

26. Grimshaw K, Maskell J, Oliver E et al. Introduction of complementary Foods and the Relationship to Food Allergy. Pediatrics. 2013; 132: e1529-e1538.

27. Aronsson C, Lee H, Liu E et al. Age at gluten Introduction and Risk of celiac disease. Pediatrics. 2015; 135: 239-245.

28. Hogen $E$, Rosen A, Auricchio R et al. The preventCD Study design: towards new strategies for the prevention of coeliac disease. Eur J Gastroenterol Hepatol. 2010; 22: 1424-30.

29. Welander A, Montgomery S, Ludvigsson J, Ludvigsson J. Breast-feeding Duration and Gluten Introduction Among Mothers with Celiac Disease. JPGN. 2014; 59: 89-92.

30. Lionetti $E$, Castellaneta S, Francavilla $R$ et al. Introduction of Gluten, HLA status, and the Risk of Celiac Disease in Children. N Engl J Med. 2014; 371: 1295-303.

31. Pawankar R, Canonica GW, Holgate ST, Lockey RF. WAO white book on allergy 2011-2012: executive summary. Milwaukee: World Allergy Organization, 2011.

32. Fiocchi A, Pawankar R, Cuello-Garcia C et al. World Allergy Organization-McMaster University Guidelines for Allergic Disease Prevention (GLAD-P): Probiotics. World Allergy Organ J. 2015; 27;8(1):4. doi: 10.1186/s40413015-0055-2. eCollection 2015.

33. Greer FR, Sicherer SH, Burks AW. American Academy of Pediatrics Committee on Nutrition; American Academy of Pediatrics Section on Allergy and Immunology. Effects of early nutritional interventions on the development of atopic disease in infants and children: the role of maternal dietary restriction, breastfeeding, timing of introduction of complementary foods, and hydrolyzed formulas. Pediatrics 2008;121:183-91.

34. Fälth-Magnusson K, Kjellman NI. Allergy prevention by maternal elimination diet during late pregnancy - a 5-year follow-up of a randomized study. J Allergy Clin Immunol. 1992;89:709-13.

35. Lilja G, Dannaeus A, Foucard T, Graff-Lonnevig V, Johansson SG, Oman $H$. Effects of maternal diet during late pregnancy and lactation on the development of IgE and 
egg- and milk-specific IgE and IgG antibodies in infants. Clin Exp Allergy 1991;21:195-202.

36. Katz $Y$, Rajuan $N$, Goldberg $M R$ et al. Early exposure to cow's milk protein is protective against IgE-mediated cow's milk protein allergy. J Allergy Clin Immunol. 2010; 126: 77-82.
37. Du Toit $G$, Katz $Y$, Sasieni $P$ et al. Early consumption of peanuts in infancy is associated with a low prevalence of peanut allergy. J Allergy Clin Immunol. 2008; 122: 984-91.

38. Rodrit C, Frei $R$, Depner $M$ et al. Increased food Diversity in the first year of life is inversely associated with allergic disease. J Allergy Clin Immunol. 2014; 133: 1056-64. 